\title{
Studying Conceptual Modeling \\ Processes: A Modeling Tool, Research \\ Observatory, and Multimodal Observation Setup
}

\author{
Stefan Strecker, Kristina Rosenthal, and Benjamin Ternes
}

\begin{abstract}
What do (non-)experienced modelers reason while conceptual modeling and how do they arrive at modeling decisions, which modeling and learning difficulties do they face and why, and how do they overcome these difficulties by tailored modeling tool support are questions of relevance and importance to practicing modelers and, likewise, to conceptual modeling research. For the past 7 years, we have been designing, developing, and evaluating a modeling tool integrating a research observatory aimed at studying individual modeling processes online, in the field, and under laboratory conditions - to contribute to a richer understanding of modeler reasoning and decision-making, to identify common modeling and learning difficulties, and, ultimately, to design tool support to mitigate difficulties and to improve assistance for (non-)experienced modelers. We present an overview of the modeling observatory and of a corresponding multimodal observation setup.
\end{abstract}

\section{Conceptual Models and Conceptual Modeling}

Conceptual models enable and empower us to shape the digital transformation of organizations - of small and medium businesses, large corporations, public administration, unions, clubs, and associations of all kinds. Without conceptual models, e.g., data models, object models, business process models, and more advanced (enterprise) models, of such enterprises and their social action systems in co-action with their computer information systems, we cannot understand the complex human-computer interactions that concern us and cannot fully seize their potential to address the problems of modern society and economy (Frank and Strecker 2021).

\footnotetext{
S. Strecker $(\bowtie) \cdot$ K. Rosenthal $\cdot$ B. Ternes

FernUniversität in Hagen, Enterprise Modelling Research Group, Lehrstuhl für

Betriebswirtschaftslehre, insbes. Entwicklung von Informationssystemen, Fakultät für

Wirtschaftswissenschaft, Hagen, Germany

e-mail: stefan.strecker@fernuni-hagen.de; kristina.rosenthal@fernuni-hagen.de;

benjamin.ternes@fernuni-hagen.de
} 
Conceptual modeling — as an activity, e.g., when constructing a data model as Entity-Relationship diagram (Chen 1976)_-involves an intricate array of cognitive processes and performed actions, including goal setting, abstracting, conceptualizing, associating and contextualizing, interpreting and sense-making, evaluating and judging, anticipating and envisioning and thinking ahead, drawing and visualizing, and, in group settings, communicating, discussing, and agreeing. The activity of conceptual modeling is, therefore, at one point in time, tool-mediated and based on interacting with modeling tools supporting conceptual modelers, e.g., by way of graphical modeling editors. Performing conceptual modeling is construed as a complex task based on codified and tacit knowledge, a task that requires mastering theoretical foundations, modeling languages (such as Entity-Relationship Model, Chen 1976), modeling methods, and modeling tools, applying them to practical problems, and, while performing a modeling process, critically thinking and reflecting upon an application domain in terms of the technical languages spoken in the targeted domain - the domain's technical terminology and its use in domain-specific technical languages, their imprecision, ambiguities, and related challenges-with the intention to (re-)construct the selected aspects of organizational reality and to reshape organizational reality by (re-)presenting a new perspective and reworked terminology and language embodied and conveyed by conceptual models (Frank et al. 2014).

The process (the actual "act") of conceptual modeling has for long received limited attention in conceptual modeling research (Hoppenbrouwers et al. 2005; 2006) but has recently seen increasing interest from researchers (e.g., Bera et al. 2019, Claes et al. 2015, Pinggera et al. 2015, Serral et al. 2016, Wilmont et al. 2017). How conceptual modeling is performed by modelers, how modeling processes proceed, which modeling challenges and difficulties modelers experience and why, and how to overcome these difficulties by tailored modeling support have been subject to studies on the cognitive processes and performed actions constituting conceptual modeling (e.g., Batra and Davis 1992, Bera 2011, Chaiyasut and Shanks 1994, Srinivasan and Te'eni 1995, Venable 1996). Still, further research on conceptual modeling processes is needed to understand the reasoning of modelers and their deliberations (e.g., about modeling decisions) and whether different (idealized) types of modelers can be identified, e.g., by determining the patterns of modeling processes and/or difficulties, and whether these modeler types benefit from modeling tool support tailored to overcome their difficulties. Similarly, further research is required to study how individuals learn conceptual modeling, how their learning progresses, and what their needs are for learning support and tool assistance (Rosenthal et al. 2019). Studying progressively more and more individual modeling processes under varying conditions, i.e., online and in the field and the laboratory, promises to shed further light on these open questions and, ultimately, to enable us to design targeted (tool) support for modelers at different stages of their learning and mastering of conceptual modeling.

Against this background, we pursue a research program to contribute to a richer and more complete understanding of individual modeling processes and of the learning of conceptual modeling - aiming for the overarching research objective of 
designing, implementing, and evaluating targeted tool support for modelers at different stages of their learning and mastering of conceptual modeling. In our pursuit of this research objective, we operate on the basic assumption that modelers' individual modeling processes demand a study from multiple complementary perspectives to account for the richness and complexity of conceptual modeling as a learning and performing task - following Berger and Luckmann's inspiring insight that "the object of thought becomes progressively clearer with this accumulation of different perspectives on it" (Berger and Luckmann 1967, p. 10). Ultimately, the research program aims to progressively add to the empirical and theoretical foundation of conceptual modeling research, e.g., by identifying modeling difficulties and by developing a taxonomic theory of such difficulties as a step toward advancing the body of knowledge to offer guidance for designing and implementing targeted tailored (tool) support for conceptual modelers. As part of this research program, we configure and validate a multimodal observation setup and corresponding analysis procedure that we realize by TOOL, a web browser-based modeling tool and research observatory implementing an array of observation and analysis features.

The subsequent Sect. 2 introduces the multimodal observation setup. Section 3 demonstrates how TOOL supports the observation setup, and Sect. 4 reports on a series of laboratory observations on individual modeling processes and concludes with lessons learned.

\section{Multimodal Observation Setup}

Observing conceptual modeling processes poses methodological challenges. For example, neither the reasoning of modelers nor the corresponding deliberations on modeling decisions are immediately accessible nor directly observable. Research on conceptual modeling processes has reverted to observable aspects of modeling processes such as modelers' interactions with software tools, modelers' eye movements, or their verbalizations of their own thought processes while modeling - as verbal protocols following the "think-aloud" method (Ericsson and Simon 1993, van Someren et al. 1994). Following this trajectory, we compile and arrange multiple complementary modes of observation for a multimodal observation setup (see Fig. 1). In particular, we combine four modes of observation:

1. Audio-taping think-aloud (verbal) protocols during conceptual modeling by a subject, i.e., while working on a modeling task, to obtain insights into the subject's cognitive processes during modeling, e.g., into the modeler's reasoning and deliberations toward modeling decision and also into difficulties the modeler encounters and expresses verbally

2. Tracking modeler interactions with the modeling canvas to observe specific modeling decisions, in particular, decisions with respect to placing a new model element on the modeling canvas (e.g., a rectangular graphical symbol representing an entity type, say "Customer," in an entity-relationship diagram), to 


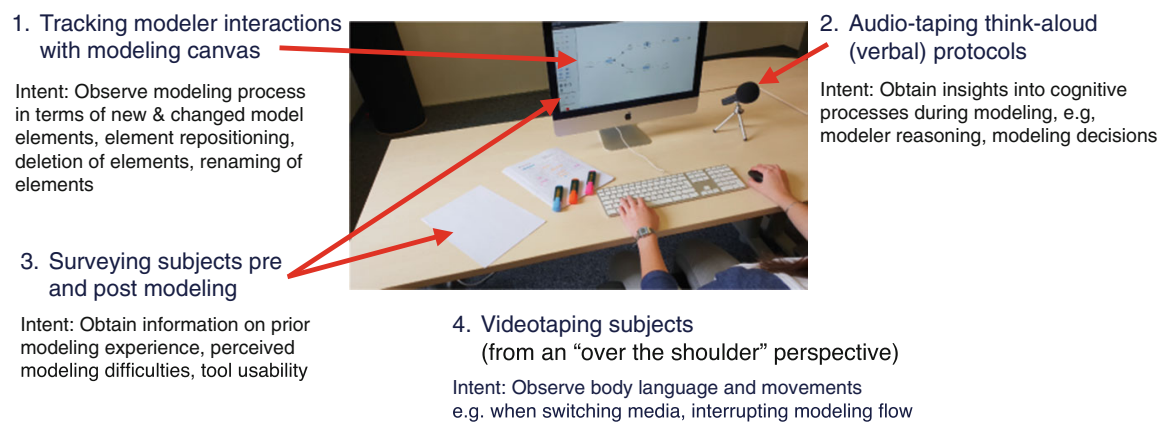

Fig. 1 Complementary modes of observation

change an existing model element, to element repositioning, to deletion of model elements, and to renaming a model element (giving it a new label)

3. Surveying subjects pre- and post-modeling to collect information on modeler demographics and to obtain self-disclosed information on modeling experience, perceived modeling difficulties, and tool usability, i.e., a post-modeling survey collects information about difficulties with using the tool's graphical editor, tool support, and overall user experience to evaluate TOOL as a design artifact

4. Videotaping subjects (e.g., from an "over-the-shoulder" perspective) to observe nonverbal cues on the individual modeling process, in particular on modeling difficulties, conveyed by body language and movement, e.g., when switching between media from the computer screen to paper and back or when interrupting the modeling flow as indicated by gestures

The main rationale for combining these modes of observation lies in their complementarity (Rosenthal and Strecker 2019): The configured setup combines observation by think-aloud protocols and video recordings with modeler-tool interaction tracking and survey data to observe a wide range of facets about individual modeling processes including identifying modeling and learning difficulties expressed verbally by the modeler during and after modeling as well as nonverbally indicated through interactions with the modeling tool and its modeling canvas as well as by body language and movements. Specifically, analyzing think-aloud protocols has shown promising results for understanding the cognitive processes of subjects working on problem-solving tasks in general and on modeling tasks in particular (e.g., Batra and Davis 1992, Srinivasan and Te'eni 1995), and we consider verbalization of thoughts as the best available means of expression for achieving insights into modelers' reasoning as our spoken language provides a rich and flexible tool to express our thinking. However, to ask subjects to think aloud is a second-best approach, warranted only because it is not possible to directly access and capture cognitive processes and, thus, modeler reasoning while modeling. Modelers may have difficulties verbalizing their reasoning while modeling (Blech et al. 2019) on principle accounts (because verbalizing one's own thoughts can be difficult) or on modeling-related accounts (e.g., because of the 
difficulty of finding the right words to express oneself). Nonetheless, among all possible alternative modes of observation, think-aloud verbalization promises the richest insight into quintessential non-directly observable cognitive processes of an individual modeling process.

Modelers' reasoning, their chain of thought, and line of modeling arguments will, still, not always be observable from think-aloud protocols alone, since nonverbal information such as modelers' movements and gestures entail important additional cues about their perception of, for instance, modeling challenges and difficulties. Hence, the configured observation setup complements think-aloud protocols with videotaping modelers to allow for additional visual clues, e.g., regarding interaction with pen and paper or modelers' (e.g., erratic) movements during modeling plus modeler-tool interactions. Additionally, subjects are surveyed about their individual modeling process before and after they work on a (controlled) modeling task. Following Berger and Luckmann, this observation setup combines multiple perspectives on modeling processes and supports mixed methods research designs in which open (narratives, verbal protocols, video recordings) and closed (more) standardized (tracking data, survey data) modes of observation are combined to obtain a more complete picture of the phenomenon under investigation (Creswell and Plano Clark 2018).

\section{Modeling Tool and Research Observatory}

The multimodal observation setup is realized based on the modeling tool TOOL integrating a research observatory designed for observing and analyzing individual modeling processes (Ternes 2017, Ternes and Strecker 2018, Ternes et al. 2019). Resulting from a multi-year design science research project, the TOOL research prototype currently implements two graphical modeling editors (see Fig. 2): (1) an editor for a didactically adapted, simplified variant of the Entity-Relationship model (ERM) for data modeling and (2) an editor for a subset of the Business Process Model and Notation (BPMN 2) for business process modeling (Frank and Strecker 2021). For studying individual modeling processes under laboratory conditions, in the field, and in online settings, TOOL integrates a research observatory adapted to the multimodal observation setup (Ternes et al. 2020a;c). The modeling observatory presently features five modes of observation (as in-browser features):

1. Recording of tink-aloud (verbal) protocols via computer audio input (in addition to external audio recording used in laboratory settings; see Fig. 1)

2. Recording of the subject's computer screen to provide an additional video recording of the modeling process

3. Timed-discrete event tracking and recording of modeler interactions with the tool's modeling canvas

4. Tracking and recording of mouse pointer movement on the modeling canvas

5. Conducting and recording on-screen pre- and post-modeling surveys of subjects (as an alternative to printed surveys) 


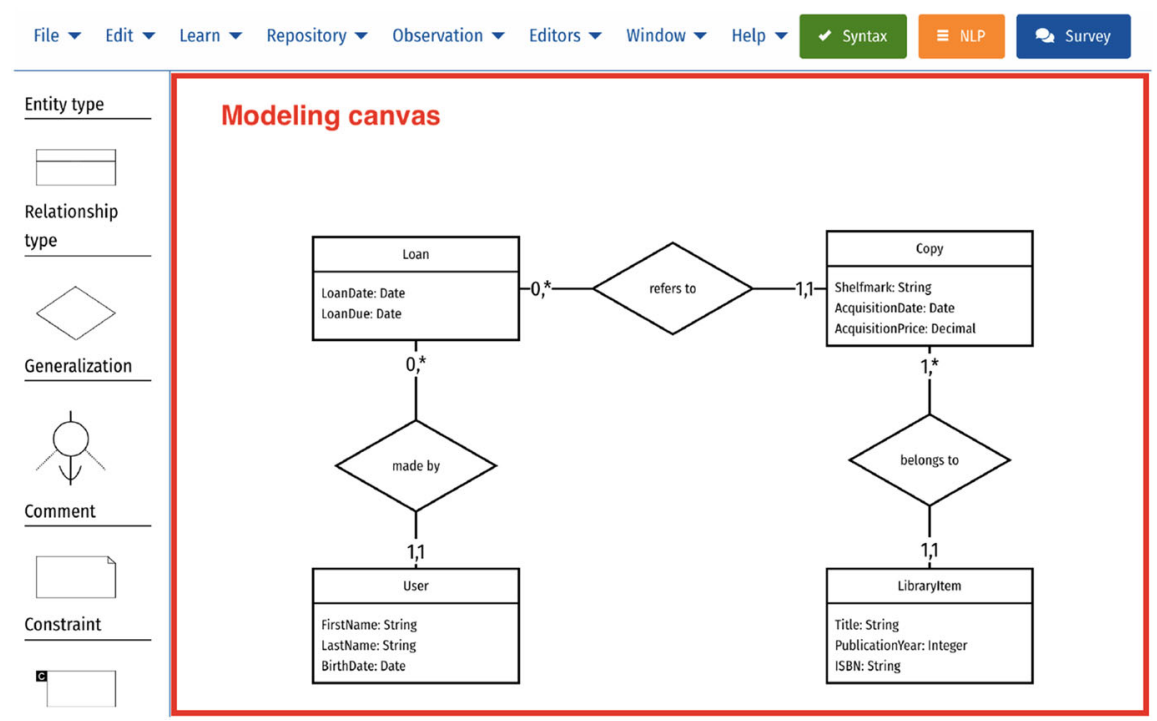

Fig. 2 Graphical editor for data modeling (with modeling canvas highlighted) (Ternes et al. 2020a)

The observatory also features data visualizations and analysis techniques for interpreting the recorded data: (a) Replay of the recorded modeler-tool interactions of a modeling process in real time or step by step, either as single replay or in comparison of up to four modeling processes shown concurrently over normalized time (see Fig. 3a): The step-by-step replay visually shows every step of the model construction, whereas the automatic replay shows model construction in real time, both allowing for additional visual inspection of the modeling process. (b) Replay with heat map overlays on mouse pointer position, dwell time, and mouse clicks (see Fig. 3b): Such heat maps allow insights into spatial areas of the modeling canvas in focus and into difficulties experienced with the modeling tool, e.g., indicated by uncontrolled mouse clicking. (c) Dot diagrams (see Fig. 3c), a data visualization inspired by PPMCharts (Claes et al. 2015) and dotted charts (Song and van der Aalst 2007): The vertical axis indicates the consecutively numbered model elements that are created (green circle), changed (blue circle), repositioned (gray), deleted (red circle), or relabeled (orange circle). The dot diagram visually differentiates the mentioned five types of specific modeling decisions (introduce new model element on canvas, change existing element, reposition an element, delete an element, relabel an element) and allows to clarify audio/video and tracking observations in cases when the observed modeling behavior is ambiguous. (d) Bar charts and related visualizations of survey data prepare for survey analysis, e.g., by visually representing results from closed-ended questions (see Fig. 3d). The visualizations and techniques $(\mathrm{a}-\mathrm{d})$ are used to further explore the observed situations identified as deviant or unclear in the audio and video protocol and to identify and clarify 


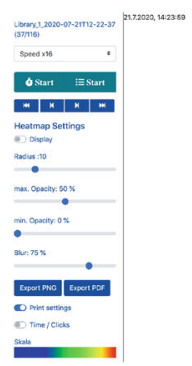

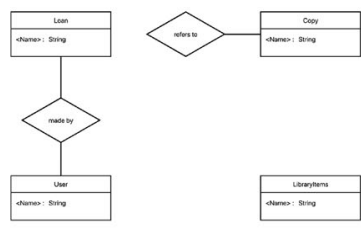

(a)

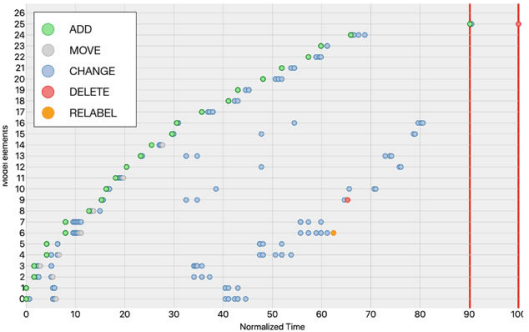

(c)

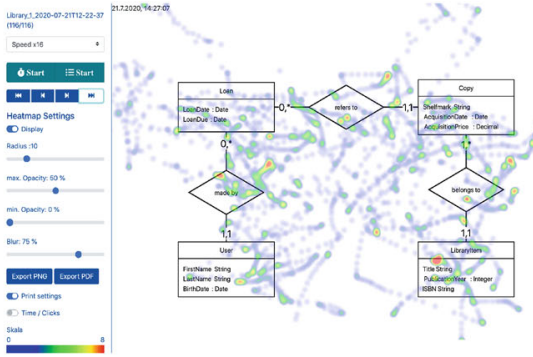

(b)

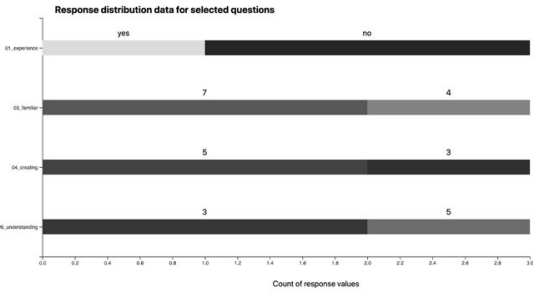

(d)

Fig. 3 Data visualization and analysis techniques (Rosenthal et al. 2020b). (a) Replay. (b) heat map, (c) dotted diagram, (d) survey results

anomalous modeling behavior by manual inspection. Additionally, the research observatory is adaptable to different research designs by selecting and combining modes of observation (1-5), by tailoring surveys, and by sequencing a corresponding observation workflow. A video demonstration of the research observatory is available at https://vimeo.com/441854796/5237d3782a.

From the outset run as a design science research project, two essential requirements drive the development of the TOOL research prototype: (1) platform independence and (2) ease of use and usability to the greatest extent economically viable. Further design considerations, requirements, and operating principles are outlined in Ternes (2017), Ternes and Strecker (2018), and Ternes et al. (2019; 2020a;c). In a nutshell, TOOL is designed and implemented as a web application with a JavaScript-driven web browser frontend and a Java EE (Enterprise Edition)-based server backend and, as a design artifact, also serves as a research laboratory for studying (web technology) software stacks and toolchains (see Fig. 4). The current working prototype sizes up to 55,000 lines of code ( $\mathrm{LoC})$ in the frontend and about $32,000 \mathrm{LoC}$ in the server backend. TOOL has been applied to an initial test series, to pilot studies, as well as in a series of research studies on individual modeling processes at the University of Hagen, Germany; the Universitat Politècnica de València, Spain; and the Katholieke Universiteit Leuven, Belgium (see next section). Moreover, TOOL has supplemented our teaching of an undergraduate course on modeling business information systems where students use it for their assignments. 


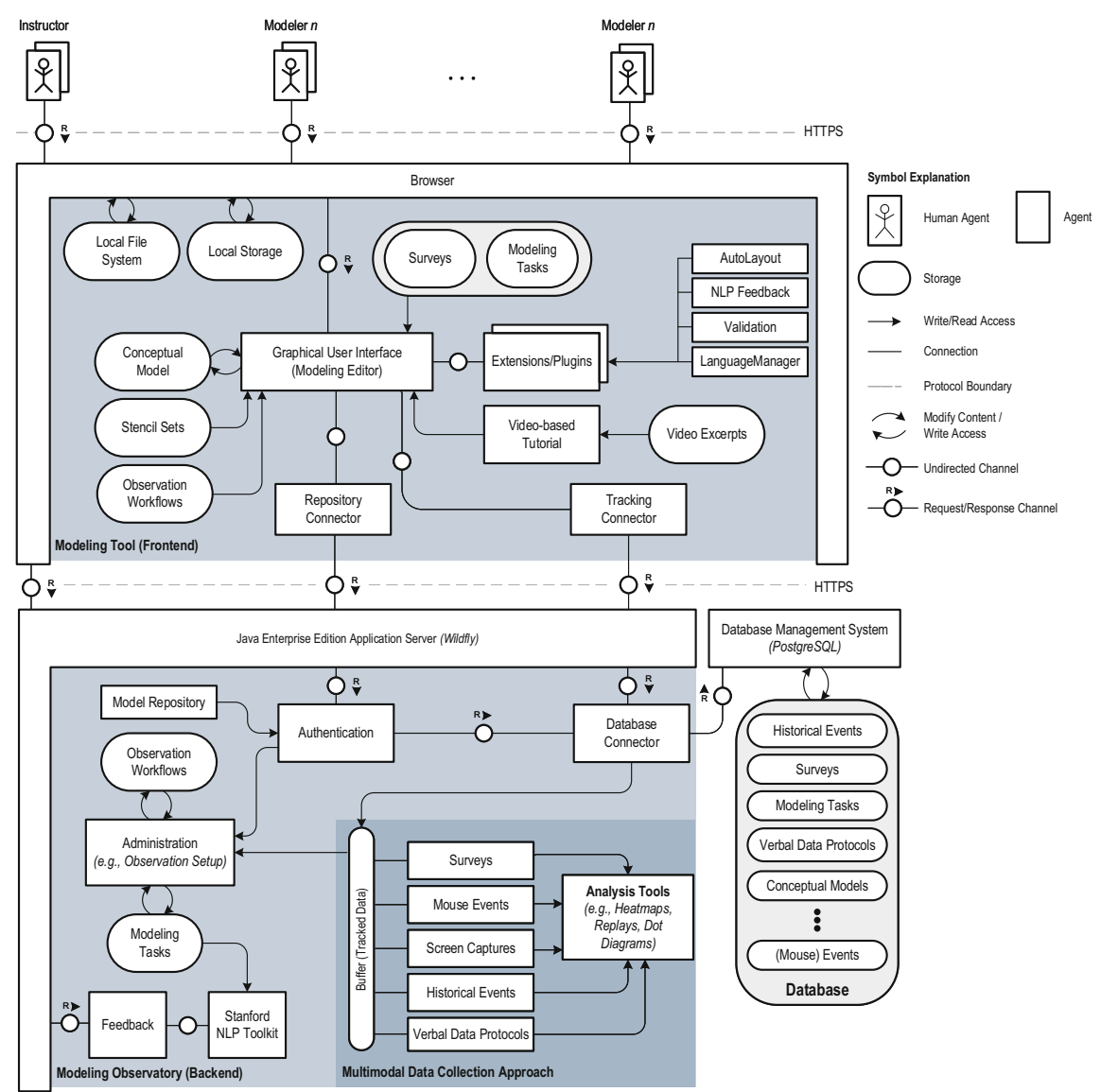

Fig. 4 TOOL software architecture overview (Enhanced from Ternes et al. 2020a)

\section{Data Integration, Data Analysis, and Lessons Learned}

A series of laboratory studies validates the multimodal observation setup in combination with the research observatory and modeling tool: For example, we have been studying individual modeling processes of experienced and non-experienced modelers to identify the modeling difficulties these modelers face while performing a data modeling task (Rosenthal and Strecker 2019, Rosenthal et al. 2020b). In a nutshell, subjects are provided with a natural language description of a data modeling task, receive instructions and an introduction to the graphical editor, and are then asked to think out loud while performing the modeling task. To achieve insights into the observed individual modeling processes, the collected data is purposefully integrated and analyzed to identify modeling difficulties and to understand modelers' reasoning: Each verbal think-aloud protocol is linked 


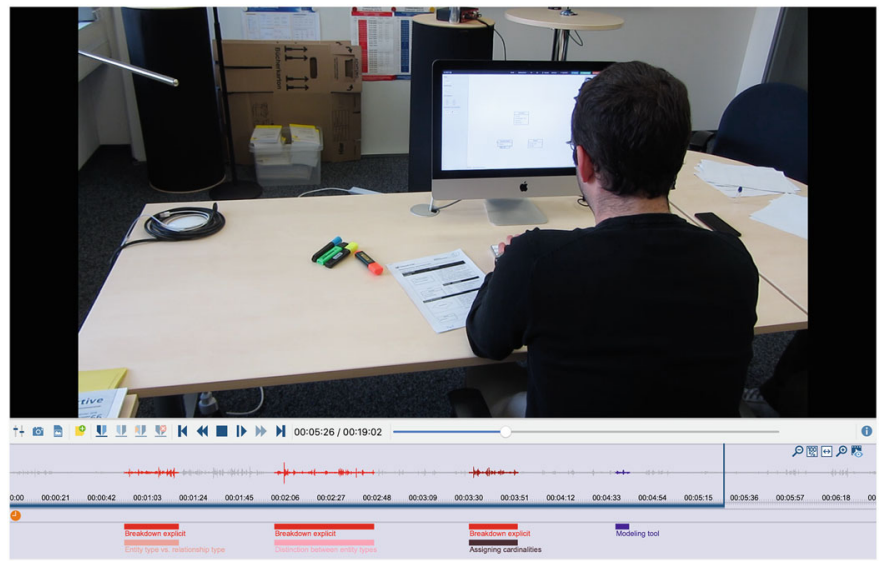

Fig. 5 Coding audiovisual protocols (Screenshot from MAXQDA, VERBI Software 2018)

and time-synchronized to the video recording to produce an audiovisual protocol comprising the data from both modes of observation. To add structure to the data, we code these videos by systematically assigning codes to video segments/clips (following, e.g., Miles et al. 2014, pp. 81f.) using a qualitative data analysis software (see Fig. 5 for an example from a pre-test). Directly coding the audiovisual protocols rather than transcribing the verbalizations allows to benefit from the complementary angles provided by the respective mode of observation. Following problem-solving research (Newell and Simon 1972), we use the concept of cognitive breakdowns (e.g., Bera 2011) to identify modeling difficulties which modelers experience while constructing a conceptual model and, hence, use cognitive breakdown as deductive code. We define a cognitive breakdown as a cognitive difficulty which a modeler experiences when constructing a conceptual model based on a natural language description (Bera 2011, p. 4)—“when a line of thought fails" (BurtonJones and Meso 2008, p. 768). Such a cognitive breakdown can manifest itself in a modeler explicitly verbalizing a difficulty while modeling or in interrupting or terminating a modeling activity, e.g., a modeling activity which is not completed, but instead the modeler switches to another activity (Bera 2011, p. 4). Segments in which a subject encounters a difficulty or an obstacle are initially marked with the code "cognitive breakdown," i.e., when the subject explicitly verbalizes a difficulty experienced during modeling or when the subject interrupts or terminates a modeling activity. This code is complemented with codes generally anticipated in think-aloud protocols as, e.g., talking about non-task-related issues, evaluation of the task at a meta-level, silent periods, and actions outside of the modeling tool (following, e.g., van Someren et al. 1994, p. 122). During coding, the coding scheme is complemented with emerging codes and sub-codes-allowing for refinements according to the actual behavior exhibited in the modeling processes. We supplement coding audiovisual protocols with analyzing timed modeler-tool interactions allowing us to identify peculiar situations. Segments identified as unclear in the 
videos are submitted for closer inspection by analyzing the recorded modeler-tool interactions in the respective time period to better understand the observed situation and to decide on assigning a code: Dot diagrams visualizing modeling processes and replays of model construction are used for further exploring situations identified as ambiguous or deviant in the audiovisual protocols. Vice versa, anomalous data in the recorded modeler-tool interactions is identified and further investigated through analyzing audiovisual protocols. Data integration is taken one step further by reviewing the pre- and post-modeling surveys and, thus, by supplementing another mode of observation (self-disclosed by the observed modeler). This coding step proved valuable especially as the individually perceived difficulties serve as indication for closer inspecting and deciding on assigning a code in the audiovisual protocols. We provide a more detailed description of the data analysis strategy and coding steps in Rosenthal and Strecker (2019, pp. 8-10).

Observations on individual modeling processes we performed so far led us to identify ten types of modeling difficulties the observed subjects faced while performing the data modeling task: The types of difficulties relate to different aspects of constructing conceptual data models, i.e., entity types, relationship types, attributes, and cardinalities. The majority of the difficulties encountered by the participants relate to modeling relationship types, i.e., deciding between modeling an entity type or a relationship type, developing sensible identifiers for relationship types, and determining cardinalities (Rosenthal and Strecker 2019, Rosenthal et al. 2020a). To overcome these modeling difficulties when formulating sensible identifiers (Breuker et al. 2016), we presently research and develop extensions to TOOL for automated modeler assistance based on natural language processing (NLP) (Manning et al. 2014) to provide tool-generated suggestions on labeling model elements at modeling time. To the best of our knowledge, this design science research is among the first to integrate a web-based data modeling tool with NLP to automatically process an arbitrary natural language description of a modeling task (in terms of its morphological structure) to identify words and phrases as suggestions for labels for model elements (Ternes et al. 2020b).

Conducting studies with experienced and non-experienced modelers demonstrates that the multimodal observation setup in combination with the research observatory opens entirely new paths for insights into conceptual modeling processes. Analyzing the collected data exemplifies that only the integration of the complementary observation modes allowed us to identify a wide range of modeling difficulties by identifying cognitive breakdowns and also to reason about the cause and type of difficulty observed: Supplementing the analysis of audiovisual protocols with analyzing tracked modeler-tool interactions enabled us to better understand ambiguous situations and to identify further deviant interactions, while reviewing the post-modeling survey proved especially valuable as the perceived difficulties served as indication for closer inspection of audiovisual protocols. Hence, our current findings suggest that combining multiple modes of observation to study individual modeling processes contributes to achieving a richer and more complete understanding of modeling processes and modeling difficulties encountered during model construction. Informed by these initial insights, we are preparing for further 
small-scale and large-scale studies aimed at deepening and substantiating our understanding of modeling difficulties and identifying patterns of modeling processes.

\section{References}

Batra D, Davis JG (1992) Conceptual data modelling in database design: Similarities and differences between expert and novice designers. International Journal of Man-Machine Studies 37(1):83-101

Bera P (2011) Situations That Affect Modelers' Cognitive Difficulties: An Empirical Assessment. In: Sambamurthy V, Tanniru M (eds) Proceedings of the 17th Americas Conference on Information Systems, AMCIS 2011, Detroit, Michigan, USA, August 4-8, 2011

Bera P, Soffer P, Parsons J (2019) Using Eye Tracking to Expose Cognitive Processes in Understanding Conceptual Models. MIS Quarterly 43(4)

Berger PL, Luckmann T (1967) The Social Construction of Reality. Anchor Books, New York, NY, reprint of the 1966 original published by Doubleday, Garden City, NJ

Blech C, Gaschler R, Bilalić M (2019) Why do people fail to see simple solutions? Using thinkaloud protocols to uncover the mechanism behind the Einstellung (mental set) effect. Thinking \& Reasoning

Breuker D, Matzner M, Delfmann P, Becker J (2016) Comprehensible predictive models for business processes. MIS Quarterly 40(4):1009-1034

Burton-Jones A, Meso P (2008) The Effects of Decomposition Quality and Multiple Forms of Information on Novices' Understanding of a Domain from a Conceptual Model. Journal of the Association for Information Systems 9(12):748-802

Chaiyasut P, Shanks GG (1994) Conceptual data modeling process: A study of novice and expert data modellers. In: Halpin TA, Meersman R (eds) Proceedings of the 1st International Conference on Object-Role Modeling, ORM-1, Magnetic Island, Australia, 4-6 July 1994, pp 310-323

Chen PPS (1976) The entity-relationship model—-toward a unified view of data. ACM Transactions on Database Systems 1(1):9-36, https://doi.org/10.1145/320434.320440

Claes J, Vanderfeesten I, Pinggera J, Reijers HA, Weber B, Poels G (2015) A visual analysis of the process of process modeling. Information Systems and e-Business Management 13(1):147-190

Creswell JW, Plano Clark VL (2018) Designing and Conducting Mixed Methods Research, 3rd edn. Sage, Los Angeles, CA

Ericsson KA, Simon HA (1993) Protocol analysis: Verbal reports as data, 2nd edn. MIT Press, Cambridge, MA

Frank U, Strecker S (2021) Modellierung betrieblicher Informationssysteme : Einführung, theoretische Grundlagen und praktische Anwendungen. DeGruyter, Berlin, in preparation

Frank U, Strecker S, Fettke P, vom Brocke J, Becker J, Sinz EJ (2014) The Research Field "Modeling Business Information Systems" : Current Challenges and Elements of a Future Research Agenda. Business \& Information Systems Engineering 6(1):39-43

Hoppenbrouwers SJBA, Proper HA, van der Weide TP (2005) A fundamental view on the process of conceptual modeling. In: Delcambre L, Kop C, Mayr HC, Mylopoulos J, Pastor O (eds) 24th International Conference on Conceptual Modeling (ER). Lecture Notes in Computer Science, Springer, Berlin, Heidelberg, vol 3716, pp 128-143

Hoppenbrouwers SJBA, Lindeman L, Proper HA (2006) Capturing modeling processes - towards the MoDial modeling laboratory. In: Meersman R, Tari Z, Herrero P (eds) On the Move to Meaningful Internet Systems 2006: OTM 2006 Workshops. Lecture Notes in Computer Science, Springer, Berlin, Heidelberg, vol 4278, pp 1242-1252 
Manning CD, Surdeanu M, Bauer J, Finkel JR, Bethard S, McClosky D (2014) The Stanford CoreNLP natural language processing toolkit. In: Proceedings of the 52nd Annual Meeting of the Association for Computational Linguistics, ACL 2014, June 22-27, 2014, Baltimore, MD, USA, System Demonstrations, pp 55-60

Miles MB, Huberman AM, Saldaña J (2014) Qualitative Data Analysis: A Methods Sourcebook, 3rd edn. Sage, Thousand Oaks, CA

Newell A, Simon HA (1972) Human problem solving. Prentice-Hall, Englewood Cliffs, NJ

Pinggera J, Soffer P, Fahland D, Weidlich M, Zugal S, Weber B, Reijers HA, Mendling J (2015) Styles in business process modeling: an exploration and a model. Software \& Systems Modeling 14(3): 1055-1080

Rosenthal K, Strecker S (2019) Toward a Taxonomy of Modeling Difficulties: A Multi-Modal Study on Individual Modeling Processes. In: Krcmar H, Fedorowicz J, Boh WF, Leimeister JM, Wattal S (eds) Proceedings of the 40th International Conference on Information Systems, ICIS 2019, Munich, Germany, December 15-18, 2019

Rosenthal K, Ternes B, Strecker S (2019) Learning Conceptual Modeling: Structuring Overview, Research Themes and Paths for Future Research. In: vom Brocke J, Gregor S, Müller O (eds) 27th European Conference on Information Systems, ECIS 2019, Stockholm and Uppsala, Sweden, June 8-14, 2019

Rosenthal K, Strecker S, Pastor O (2020a) Modeling Difficulties in Data Modeling - Similarities and Differences Between Experienced and Non-experienced Modelers. In: Dobbie G, Frank U, Kappel G, Liddle SW, Mayr HC (eds) Proceedings of the 39th International Conference, ER 2020, Vienna, Austria, November 3-6, 2020, Springer, pp 501-511

Rosenthal K, Ternes B, Strecker S (2020b) Understanding individual processes of conceptual modeling: A multi-modal observation and data generation approach. In: Bork D, Karagiannis D, Mayr HC (eds) Proceedings of the Modellierung 2020, Vienna, Austria, February 19-21, 2020, Gesellschaft für Informatik e.V., Lecture Notes in Informatics, vol P-302, pp 77-92

Serral E, De Weerdt J, Sedrakyan G, Snoeck M (2016) Automating Immediate and Personalized Feedback: Taking Conceptual Modelling Education to a Next Level. In: 10th International Conference on Research Challenges in Information Science (RCIS), IEEE, Grenoble, France, pp 1-6

van Someren MW, Barnard YF, Sandberg JAC (1994) The Think Aloud Method: A Practical Guide to Modelling Cognitive Processes. Academic Press, London

Song M, van der Aalst WMP (2007) Supporting process mining by showing events at a glance. In: 17th Annual Workshop on Information Technologies and Systems (WITS), Montreal, Canada, pp 139-145

Srinivasan A, Te'eni D (1995) Modeling as Constrained Problem Solving: An Empirical Study of the Data Modeling Process. Management Science 41(3):419-434, https://doi.org/10.1287/ mnsc.41.3.419

Ternes B (2017) Design and evaluation of a web-based modeling platform to support the learning of conceptual modeling and of studying the corresponding learning processes. In: Gulden J, Nurcan S, Reinhartz-Berger I, Guédria W, Bera P, Guerreiro S, Fellmann M, Weidlich M (eds) Joint Proceedings of the Radar tracks at the 18th BPMDS, and the 22nd EMMSAD, and the 8th International Workshop on Enterprise Modeling and Information Systems Architectures (EMISA) co-located with the 29th International Conference on Advanced Information Systems Engineering 2017 (CAiSE 2017), Essen, Germany, June 12-13, 2017, CEUR-WS.org, CEUR Workshop Proceedings, vol 1859, pp 138-142

Ternes B, Strecker S (2018) A web-based modeling tool for studying the learning of conceptual modeling. In: Schaefer I, Karagiannis D, Vogelsang A, Méndez D, Seidl C (eds) Modellierung 2018, Braunschweig, Germany, February 21-23, 2020, Gesellschaft für Informatik e.V., Lecture Notes in Informatics, vol P-280, pp 325-328

Ternes B, Strecker S, Rosenthal K, Barth H (2019) A browser-based modeling tool for studying the learning of conceptual modeling based on a multi-modal data collection approach. In: Ludwig T, Pipek V (eds) 14th International Conference on Wirtschaftsinformatik (WI 2019), Siegen, Germany, February 24-27, 2019, pp 1984-1988 
Ternes B, Rosenthal K, Barth H, Strecker S (2020a) TOOL - modeling observatory \& tool: An update. In: Michael J, Bork D, Fill H, Fettke P, Karagiannis D, Köpke J, Koschmider A, Mayr HC, Rehse J, Reimer U, Striewe M, Tropmann-Frick M, Ullrich M (eds) Companion Proceedings of Modellierung 2020 Short, Workshop and Tools \& Demo Papers co-located with Modellierung 2020, Vienna, Austria, February 19-21, 2020, CEUR-WS.org, CEUR Workshop Proceedings, vol 2542, pp 198-202

Ternes B, Rosenthal K, Strecker S (2020b) Automated Assistance of Data Modelers: A HeuristicsBased Natural Language Processing Approach. Internal Working Paper. Enterprise Modelling Research Group, University of Hagen, Germany

Ternes B, Rosenthal K, Strecker S, Bartels J (2020c) TOOL-A Modeling Observatory \& Tool for Studying Individual Modeling Processes. In: Michael J, Torres V (eds) ER Forum, Demo and Posters 2020 co-located with 39th International Conference on Conceptual Modeling (ER 2020), Vienna, Austria, November 3-6, 2020, CEUR-WS.org, CEUR Workshop Proceedings, vol 2716, pp 178-182

Venable JR (1996) Teaching novice conceptual data modellers to become experts. In: International Conference Software Engineering: Education and Practice, IEEE, Dunedin, New Zealand, pp $50-56$

VERBI Software (2018) MAXQDA Standard 12. https://www.maxqda.com, accessed: 2020-11-12 Wilmont I, Hoppenbrouwers S, Barendsen E (2017) An Observation Method for Behavioral Analysis of Collaborative Modeling Skills. In: Metzger A, Persson A (eds) Advanced Information Systems Engineering Workshops. CAiSE 2017. Lecture Notes in Business Information Processing, vol 286, Springer, Cham, pp 59-71

Open Access This chapter is licensed under the terms of the Creative Commons Attribution 4.0 International License (http://creativecommons.org/licenses/by/4.0/), which permits use, sharing, adaptation, distribution and reproduction in any medium or format, as long as you give appropriate credit to the original author(s) and the source, provide a link to the Creative Commons licence and indicate if changes were made.

The images or other third party material in this chapter are included in the chapter's Creative Commons licence, unless indicated otherwise in a credit line to the material. If material is not included in the chapter's Creative Commons licence and your intended use is not permitted by statutory regulation or exceeds the permitted use, you will need to obtain permission directly from the copyright holder. 